\title{
Neurosecretion in the Test Tube
}

Biochemists delight in converting the complex cellular structure of animal tissue into pipettable liquid soups by means of homogenizers, blenders and other instruments of controlled destruction. The application of such procedures to the extraordinarily heterogeneous and complex architecture of brain tissue has been known to make neuroanatomists wince, and others with some understanding of the nature of the insult thus inflicted may understandably question the wisdom of such destructive attacks.

It has been apparent for the past ten years, however, that some very fortunate and useful things happen to brain tissue during homogenization. Whittaker and his colleagues and De Robertis and his group opened up an important new approach to neurochemical studies in the early 1960s by their discovery of synaptosome particles in brain homogenates. Large numbers of such particles, representing membrane-bound structures, derived from the pinching-off and resealing of nerve endings, form when brain tissue is homogenized in an isotonic sucrose medium. Synaptosomes represent an enriched source of neurotransmitters and their related enzymes and storage mechanisms, and they can be separated from other subcellular particles present in the homogenates by density gradient centrifugation procedures.

During the past few years it has, furthermore, been found that isolated synaptosomes are capable of maintaining a surprising variety of metabolic and neurotransmitterrelated functions in vitro. Thus, Bradford (Brain Res., 19. 239; 1970; and in earlier studies) showed that synaptosomes are capable of sustaining high rates of respiration when incubated in glucose-containing buffered artificial salt media, and that a selective release of neurotransmitter amines and amino-acids could be evoked from such particles by electrical stimuli, or by exposure to high external potassium concentrations to cause depolarization of their membranes. Snyder and his colleagues (Logan and Snyder, Nature, 234, 297; 1971) have shown that synaptosome particles offer a useful in vitro system for studies of the various specific high affinity uptake systems for amines and amino-acid transmitters that exist in the different categories of neurone in the brain.

Edwardson, Bennett and Bradford describe, on page 554 of this issue of Nature, an important new application of synaptosomes as a model for studies of neurosecretion in vitro. It has previously been established that homogenates of brain tissue dissected from the base of the hypothalamus contain a special category of synaptosome representing the pinched-off terminals of the neurosecretory neurones present in this brain structure. Such synaptosomes contain many, and probably all, of the various different hypothalamic "releasing factor" peptides, which play a key part in the nervous control of pituitary function and the synaptosome preparations also contain vasopressin and oxytocin. Edwardson et al. show that synaptosome preparations of this type can be used as model systems for studying the release of these peptides in the test tube.

A synaptosome fraction was prepared from sheep hypothalamic tissue, and the synaptosomes were incubated in a balanced salt medium in vessels especially fitted with electrodes so that the contents could be subjected to electrical field stimulation. Alternatively the synaptosomes were stimulated by depolarizing concentrations $(55 \mathrm{mM})$ of potassium. Both types of stimulation caused a release of three peptides-corticotropin releasing factor $(\mathrm{CRF})$, vasopressin and prolactin inhibitory factor (PIF)into the incubating medium. Stimulation caused a five to ten-fold increase in the rate of release of the first two substances. The released peptides were estimated by bioassay, and also more directly by their effects on ACTH or prolactin secretion from anterior pituitary tissue incubated in vitro.

In parallel experiments it was also found that synaptosomes prepared from the sheep hypothalamic tissue selectively release the putative transmitter amino-acids glutamate, aspartate and gamma-aminobutyrate on stimulation, as previously found by Bradford (1970). Electrical stimulation resulted in the release of more than 50 per cent of the total synaptosome content of these three amino-acids into the incubating medium, whereas less than 10 per cent of the content of the non-transmitter aminoacids glutamine, serine, glycine and alanine were released.

These experiments should give cause for excitement to neuroendocrinologists and to neurochemists. It is clear that the synaptosome system offers many new possibilities for studying the complex factors which control the storage and release of hypothalamic neurosecretory peptides; processes that are difficult or impossible to study in vivo. Edwardson et al. hint that it may also be possible to study the biosynthesis of the releasing factors in this test tube system. It seems that the audacious assaults inflicted on central nervous tissues by the biochemist can sometimes and release of hypothalamic neurosecretory peptides, pay handsome dividends.-From our Neuropharmacology Correspondent.

\section{Oceunic Microeurthquakes}

DURING the past five years or so, the development of efficient portable seismograph stations and dependable, low-cost telemetry systems for transmitting seismic data continuously by radio or telephone has led to a significant increase in the information that may be derived from the study of small earthquakes. In geological studies, one of the advantages of using microearthquakes (often taken to mean shocks with $m_{\mathrm{b}}$ less than about 3 ) as opposed to earthquakes of higher magnitude is, of course, that there are a lot more of them. There are thus statistical advantages to be gained once it becomes possible to detect them efficiently and to determine their basic characteristics.

The area where microearthquakes have been pursued most vigorously is probably the western United States. In California, for example, Eaton et al. (Bull. Seismol. Soc. Amer., 60, $1151 ; 1970$; and Tectonophysics, 9, 259; 